\title{
Metabolic Engineering of Crocin Biosynthesis in Nicotiana Species
}

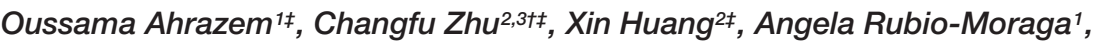 \\ Teresa Capell², Paul Christou ${ }^{2,4}$ and Lourdes Gómez-Gómez ${ }^{1 *}$
}

1 Departamento de Ciencia y Tecnología Agroforestal y Genética, Instituto Botánico, Universidad de Castilla-La Mancha, Campus Universitario, Albacete, Spain, ${ }^{2}$ Department of Plant Production and Forestry Science, University of Lleida-Agrotecnio Centre de Recerca en Agrotecnologia (CERCA) Center, Lleida, Spain, ${ }^{3}$ School of Life Sciences, Changchun Normal University, Changchun, China, ${ }^{4}$ Catalan Institute for Research and Advanced Studies (ICREA), Catalan Institute for Research and Advanced Studies, Barcelona, Spain

OPEN ACCESS

Edited by:

Barbara Molesini,

University of Verona, Italy

Reviewed by:

Luisa Bortesi,

Maastricht University, Netherlands

Guy Polturak,

John Innes Centre, United Kingdom

*Correspondence:

Lourdes Gómez-Gómez marialourdes.gomez@uclm.es

${ }^{\dagger}$ Deceased

\#These authors have contributed equally to this work

Specialty section:

This article was submitted to Plant Biotechnology, a section of the journal

Frontiers in Plant Science

Received: 24 January 2022

Accepted: 11 February 2022

Published: 08 March 2022

Citation:

Ahrazem $O$, Zhu C, Huang $X$,

Rubio-Moraga A, Capell T, Christou P and Gómez-Gómez L (2022)

Metabolic Engineering of Crocin

Biosynthesis in Nicotiana Species.

Front. Plant Sci. 13:861140.

doi: $10.3389 / \mathrm{fp} / \mathrm{s} .2022 .861140$
Crocins are high-value soluble pigments that are used as colorants and supplements, their presence in nature is extremely limited and, consequently, the high cost of these metabolites hinders their use by other sectors, such as the pharmaceutical and cosmetic industries. The carotenoid cleavage dioxygenase $2 \mathrm{~L}(\mathrm{CsCCD} 2 \mathrm{~L})$ is the key enzyme in the biosynthetic pathway of crocins in Crocus sativus. In this study, CsCCD2L was introduced into Nicotiana tabacum and Nicotiana glauca for the production of crocins. In addition, a chimeric construct containing the Brevundimonas sp. $\beta$-carotene hydroxylase (BrCrtZ), the Arabidopsis thaliana ORANGE mutant gene (AtOrMut), and CsCCD2L was also introduced into N. tabacum. Quantitative and qualitative studies on carotenoids and apocarotenoids in the transgenic plants expressing CsCCD2L alone showed higher crocin level accumulation in $N$. glauca transgenic plants, reaching almost $400 \mu \mathrm{g} / \mathrm{g}$ DW in leaves, while in N. tabacum $36 \mu \mathrm{g} / \mathrm{g}$ DW was obtained. In contrast, N. tabacum plants coexpressing CsCCD2L, BrCrtZ, and AtOrMut accumulated, 3.5fold compared to $N$. tabacum plants only expressing CsCCD2L. Crocins with three and four sugar molecules were the main molecular species in both host systems. Our results demonstrate that the production of saffron apocarotenoids is feasible in engineered Nicotiana species and establishes a basis for the development of strategies that may ultimately lead to the commercial exploitation of these valuable pigments for multiple applications.

Keywords: apocarotenoids, biotechnology, CsCCD2L, crocins, Nicotiana, saffron

\section{INTRODUCTION}

Carotenoids are an important group of natural and lipid-soluble pigments which humans cannot synthesize, thus acquiring them through diet. In humans, these molecules play multiple physiological and nutritional functions (Fiedor and Burda, 2014). Carotenoids are synthesized by plants, algae, fungi, and bacteria, displaying a yellow to red coloration, depending on the type of carotenoid and the concentration they reach in the different cells. Carotenoids are widely used as food colorants, nutraceuticals, animal feed additives, cosmetic ingredients, and health supplements (Fraser and Bramley, 2004). In all living organisms, carotenoids act as substrates to produce apocarotenoids (Ahrazem et al., 2016a). These metabolites are not simply breakdown products of carotenoids, as their functions include those of signaling and hormonal (Walter et al., 2010; 
Eroglu and Harrison, 2013; Jia et al., 2017). Apocarotenoids are present as volatile and soluble compounds. Crocins are watersoluble pigments of high commercial value, used mostly in food and to a lesser extent, in the pharmaceutical industries (Ahrazem et al., 2015). Crocins are glycosylated derivatives of the apocarotenoid crocetin and they exhibit a strong coloring capacity. In addition, crocins are powerful free radical quenchers, which is associated with the broad range of their health benefits (Nam et al., 2010; Georgiadou et al., 2012; Christodoulou et al., 2015; Bukhari et al., 2018). Interest in the therapeutic properties of crocins is increasing due to their analgesic and sedative properties (Amin and Hosseinzadeh, 2012), neurological protection, and anticancer activities (Finley and Gao, 2017; Skladnev and Johnstone, 2017). Furthermore, clinical trials indicate that crocins have a positive effect in the treatment of depression and dementia (Lopresti and Drummond, 2014; Mazidi et al., 2016). However, commercial utilization of crocins from saffron is limited due to elevated prices, a consequence of the high labor costs in harvesting and processing flower material (Ahrazem et al., 2015).

Crocus sativus is the main natural source of crocins, which accumulate at high levels during the development of the stigma and confer a dark red coloration (Moraga et al., 2009). Together with the safranal (2,6,6-trimethyl-1,3cyclohexadiene-1-carboxaldehyde), the precursor of picrocrocin ( $\beta$-D-glucopyranoside of hydroxyl- $\beta$-cyclocitral) is responsible for the flavor of the saffron spice (Tarantilis et al., 1995). In addition to Crocus, gardenia (Gardenia jasminoides) fruits are also a commercial source of crocins, but on a much lower scale, as they do not accumulate picrocrocin (Pfister et al., 1996; Moras et al., 2018). Among other plants that produce crocins, but which are not commercially exploited due to the low quantities accumulated, are Buddleja species (Liao et al., 1999). Therefore, only a few plant species can synthesize crocins, and this is because the carotenoid cleavage dioxygenases (CCDs) responsible for the production of crocins are not usually present in plants.

In Crocus, Gardenia, and Buddleja species, zeaxanthin is the precursor of crocetin (Figure 1). Cleavage of the zeaxanthin molecule at the 7,8 and $7^{\prime}, 8^{\prime}$ double bonds generates one molecule of crocetin dialdehyde and two molecules of 4-hydroxy-2,6,6trimethyl-1-cyclohexene-1-carboxaldehyde (HTCC) (Frusciante et al., 2014; Ahrazem et al., 2016c, 2017). Crocetin dialdehyde is further converted to crocetin by the action of aldehyde dehydrogenase enzymes (ALDH), and crocetin is the substrate of glucosyltransferases (UGTs) that catalyze the formation of crocins, catalyzing the transfer of glucose molecules to both ends of the crocetin molecule (Moraga et al., 2004; Nagatoshi et al., 2012; Diretto et al., 2021). The HTCC molecule is also recognized by UGTs, resulting in the formation of picrocrocin, which is further metabolized to render safranal (Figure 1; López et al., 2021). In C. sativus the CCD2 enzyme catalyzed the cleavage of zeaxanthin at 7,8; $7^{\prime}, 8^{\prime}$ double bonds (Frusciante et al., 2014). CsCCD2L is a plastidic enzyme that belongs to a new CCD subfamily only isolated from Crocus species (Ahrazem et al., 2016c), which is closely related to the CCD1 subfamily (Ahrazem et al., 2016a,b). In Buddleja davidii, and G. jasminoides, the CCD enzymes that catalyzed the same reaction belong to the CCD4 subfamily of CCDs (Ahrazem et al., 2016a; Xu et al., 2020). The Buddleja enzymes, BdCCD4.1 and BdCCD4.3, are as well plastidic enzymes expressed in flowers (Ahrazem et al., 2017).

The carotenoid biosynthetic pathway has been modified by genetic engineering in higher plants to increase the general amount of carotenoids and/or to produce specific carotenoids (Farre et al., 2014). In previous studies, CsCCD2L was transiently expressed alone and was sufficient to allow the significant crocins accumulation in tobacco plants (Diretto et al., 2019; Marti et al., 2020; López et al., 2021). In this study, we achieved a stable expression of the CsCCD2L gene alone or in combination with genes involved in the carotenoid biosynthesis and accumulation, like $\mathrm{BrCrtZ}$ and AtOrMut, to increase the availability of substrates for CsCCD2L activity. We chose the Nicotiana species as a heterologous host. The high metabolic versatility, non-food status, and high biomass production of the genus Nicotiana make this genus one of the most promising biofactories for the sustainable production of high-value metabolites (Molina-Hidalgo et al., 2021). Metabolic analyses showed the accumulation of these nutritional substances reaching in Nicotiana glauca almost $400 \mu \mathrm{g} / \mathrm{g}$ DW, which indicates that $N$. glauca is a better system than Nicotiana tabacum to produce crocins because the transgenic N. glauca plants expressing CsCCD2L alone could accumulate much higher amounts of crocins as compared with the transgenic N. tabacum plants coexpressing CsCCD2L, BrCrtZ, and AtOrMut genes.

\section{MATERIALS AND METHODS}

\section{Plant Material}

Wild-type (Wt) tobacco (N. glauca and N. tabacum cv. SR1) and transgenic plants were grown in pots with soil (Traysubstrat, Klasmann-Deilmann GmbH, Postfach 1250, 49741 Geeste, Germany) in a controlled growth chamber with a $25 / 20^{\circ} \mathrm{C}$ day/night temperature regime, a $12-\mathrm{h}$ photoperiod (mean irradiance $100 \mu \mathrm{mol} \mathrm{m} \mathrm{m}^{-2} \mathrm{~s}^{-1}$ ) and $60-90 \%$ relative humidity. The fully expanded mature leaves (the 5th and 6th leaves) were collected from five Wt and five transgenic plants for each line. All materials were frozen in liquid nitrogen and stored at $-80^{\circ} \mathrm{C}$.

After leaf sample collection, Wt and transgenic N. glauca plants were grown in a controlled growth chamber with a $25 / 20^{\circ} \mathrm{C}$ day/night temperature regime, a 12 -h photoperiod (mean irradiance $400 \mu \mathrm{mol} \mathrm{m} \mathrm{m}^{-2} \mathrm{~s}^{-1}$ ) and $60-90 \%$ relative humidity. Wt and transgenic tobacco plants were self-pollinated to obtain seeds.

\section{Vector Construction}

Two plasmids were created to evaluate their ability to engineer the saffron apocarotenoid pathway in N. glauca and N. tabacum (Supplementary Figure 1). The tobacco Ubi.U4, Arabidopsis AtUBQ10, and CaMV35S were used to drive the expression of the transgenes. The Goldenbraid strategy was followed to construct the vectors (Sarrion-Perdigones et al., 2013, 2014). Briefly, the complete open reading frames of CsCCD2L, BrCrtZ, and AtOrMut were domesticated by removing BsmBI and $B s a \mathrm{I}$ restriction sites in the original sequence using the 


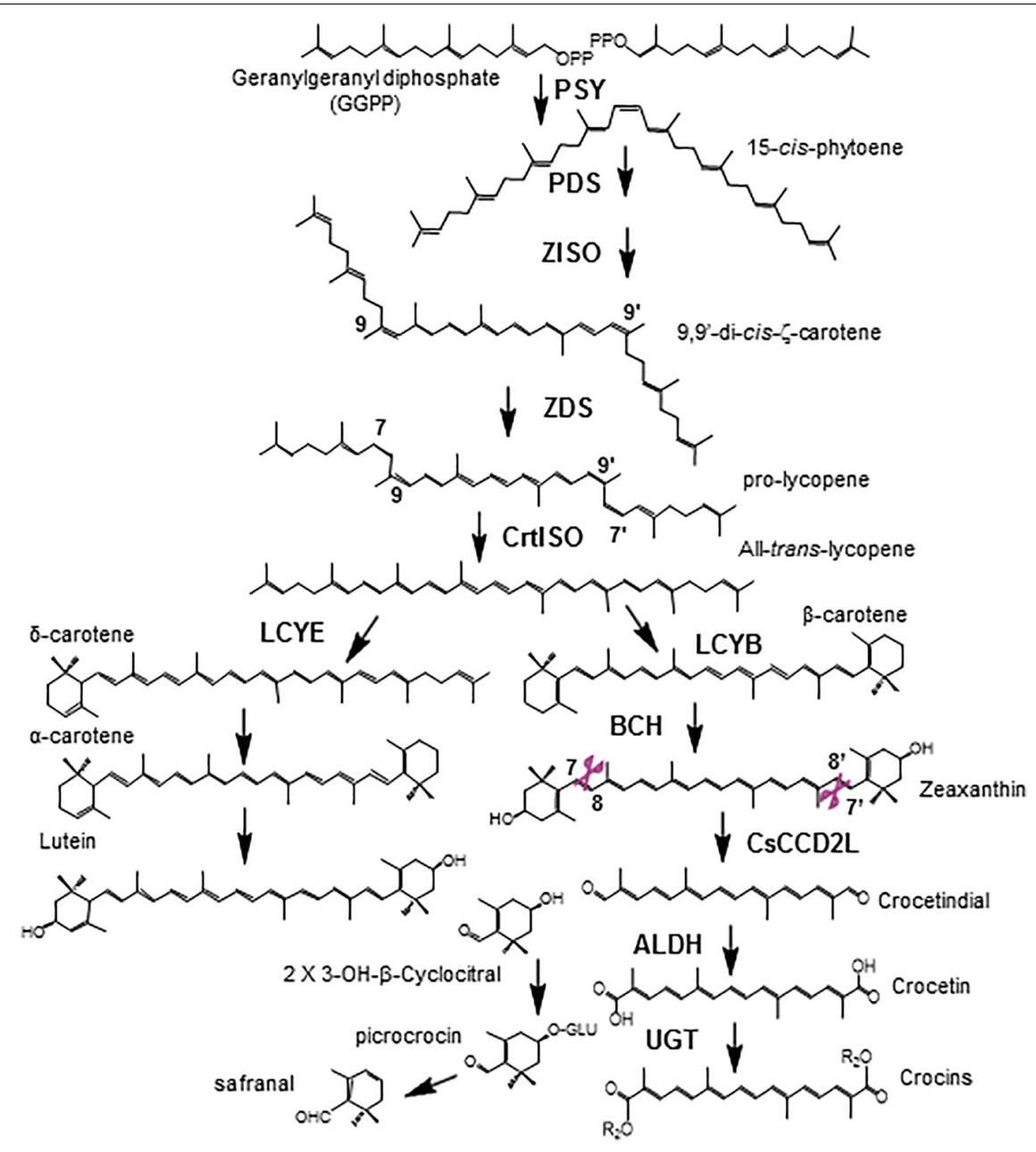

FIGURE 1 | Biosynthetic pathway of crocins in saffron. PSY, phytoene synthase; PDS, phytoene desaturase; Z-ISO, $\zeta$-carotene isomerase; ZDS, $\zeta$-carotene desaturase; CrtISO, carotene isomerase; LCYB, $\beta$-lycopene cyclase; LCYE, $\varepsilon$-lycopene cyclase; BCH, $\beta$-carotene hydroxylase; ALDH, aldehyde dehydrogenase. 3-OH- $\beta$-cyclocitral also known as HTCC (hydroxy-2,6,6-trimethyl-1-cyclohexen-1-carboxaldehyde). Cleavage positions recognized by CsCCD2L are shown in purple.

primers listed in Supplementary Table 1 . The products were cloned in the level 0 vector pUPD2 of the Goldenbraid modular cloning system. The resulting plasmids pUPD2CsCCD2L, pUPD2-BrCrtZ, and pUPD2-AtOrMut were then used to construct 2 recombinant binary vectors as follows: pDGB3 21 [p35S:CCD2L:T35S-pNos:Hyg:T35S] and pDGB3 $\alpha 1$ [p35S:CCD2L:T35S-pNos:Hyg:T35S-pTUBI4U:BrCrtZ:T35S-pA tUBQ10:AtOrMut:T35S].

\section{Transformation of Agrobacterium and Tobacco}

Agrobacterium tumefaciens strain LBA4404 was used for the above binary plasmid transformation. This strain was grown on YEB medium supplemented with $50 \mu \mathrm{g} / \mathrm{ml}$ rifampicin and $50 \mu \mathrm{g} / \mathrm{ml}$ gentamicin final concentrations for 2 days at $28^{\circ} \mathrm{C}$. The plasmids pDGB

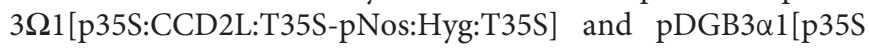
:CCD2L:T35S-pNos:Hyg:T35S-pTUB
I4U:BrCrtZ:T35S-pAtUBQ10:AtOrMut:T35S] were introduced into the bacteria by electroporation. Clones were selected on YEB agar plates containing $100 \mu \mathrm{g} / \mathrm{ml}$ rifampicin, $50 \mu \mathrm{g} / \mathrm{ml}$ spectinomycin or $50 \mu \mathrm{g} / \mathrm{ml}$ kanamycin, and 25 $\mu \mathrm{g} / \mathrm{ml}$ gentamicin.

Agrobacterium-mediated transformation of both tobacco species (N. glauca and N. tabacum) was performed according to the leaf disc method of Horsch et al. (1985). For the selection of transformants, the antibiotic hygromycin B at a final concentration of $50 \mu \mathrm{g} / \mathrm{ml}$ was used.

\section{Carotenoid and Apocarotenoid Extraction and Quantification}

Metabolite extraction and analysis differed depending on the nature of the metabolites. Polar and apolar metabolites were extracted from 50 to $5 \mathrm{mg}$ of lyophilized leaves, respectively. For the analysis of polar metabolites, leaves were extracted in cold 50\% methanol. The soluble fractions were analyzed by a 
high-performance liquid chromatography-diode array detector (HPLC-DAD) as previously described (Marti et al., 2020). The insoluble fractions (carotenoids) were extracted with 1:2 cold extraction solvents (50:50 methanol: $\mathrm{CHCl}_{3}$ ), and analyzed by HPLC-DAD as previously described (Marti et al., 2020). Pigments were quantified by integrating peak areas that were converted to concentrations by comparison with the standards and as reported before (Diretto et al., 2019). All the samples were analyzed in triplicate.

\section{mRNA Expression}

Total RNA was isolated using RNeasy Plant Mini Kit (Qiagen, Germany), after DNase treatment, first-strand cDNA synthesis was performed using the SuperScript First-Strand Synthesis System (Takara, Otsu, Japan) primed with oligo (dT)18 following the manufacturer's instructions. qRT-PCR was performed with a Fluorescent Quantitative PCR Detector (Apply Biosystems). SYBR Green real-time PCR Master Mix (Promega) was used. The amplified DNA fragments for each gene were confirmed by Sanger sequencing. The actin gene was used as a reference gene. qRT-PCR products were assessed by melting curve and gel electrophoresis to ensure the specificity of the amplification in the reactions. Three technical replicates were carried out for each biological sample. Conditions for qRT-PCR cycling were $95^{\circ} \mathrm{C}$ for $3 \mathrm{~min}, 95^{\circ} \mathrm{C}$ for $20 \mathrm{~s}, 60^{\circ} \mathrm{C}$ for $20 \mathrm{~s}, 40$ cycles. The relative expression level of each gene was calculated using the $2^{-\Delta} \Delta$ $\mathrm{Cq}$ method. Primers used in the qRT-PCR analysis are listed in Supplementary Table 1.

\section{RESULTS}

\section{Introduction of CsCCD2L in the Nicotiana glauca and Nicotiana tabacum Genomes}

We set out to produce crocins in two different host tobacco species $N$. tabacum and N. glauca. For this purpose, two binary vectors were constructed using the Goldenbraid strategy (Sarrion-Perdigones et al., 2013, 2014). The first construct contained only CsCCD2L transgene under the control of the cauliflower mosaic virus (CaMV) 35S promoter together with the hygromycin phosphotransferase selectable marker $(h p t)$ gene (Supplementary Figure 1). The second construct carried three genes: CsCCD2L, BrCrtZ, and AtOrMut is driven by the CaMV35S promoter, the tobacco polyubiquitin Ubi.U4, and the Arabidopsis AtUBQ10 promoter, respectively, together with the hygromycin gene as a selection marker (Supplementary Figure 1).

To produce crocins in stably transgenic plants, two tobacco species ( $N$. tabacum and N. glauca) were transformed with CsCCD2L under the control of the CaMV35S promoter (Supplementary Figure 1). Seventy-four putative transgenic N. tabacum plants (T0) were obtained. Four out of these seventyfour plants showed a bleaching phenotype (Supplementary Figure 2). All plants were screened for apocarotenoid analysis in green leaves by HPLC, and all these transgenic plants accumulated crocins. Wt and all the crocin accumulating transgenic N. tabacum T0 plants were fertile and self-pollinated to
A
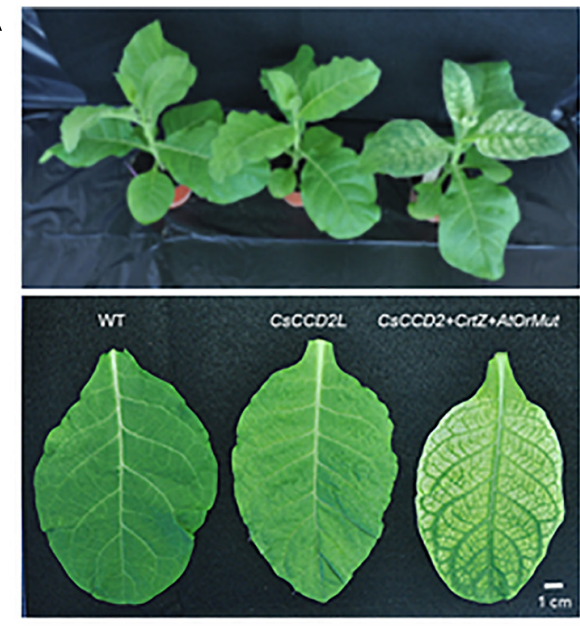

B
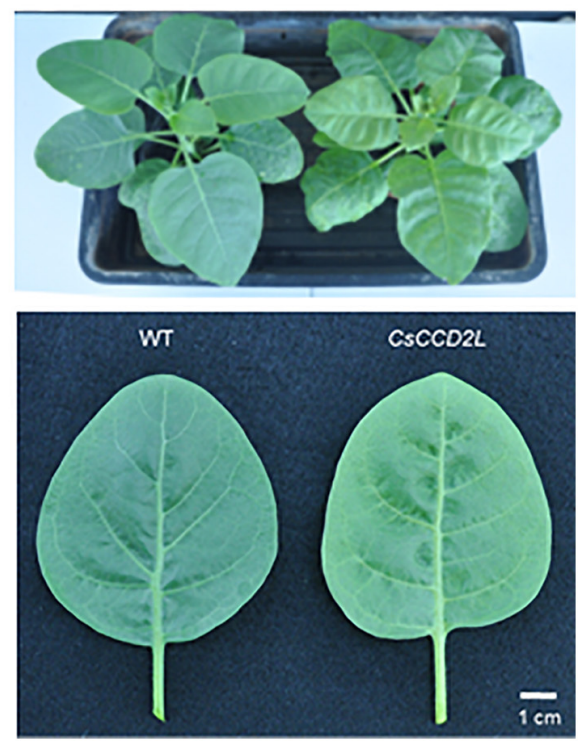

FIGURE 2 | The phenotypes of tobacco plants. (A) Plants and sixth leaves from Nicotiana tabacum wild-type (Wt) control and T2 homozygous lines expressing CsCCD2L or coexpressing CsCCD2L + BrCrtZ + AtOrMut genes. (B) Plants and sixth leaves from N. glauca Wt control and T1 hygromycin resistant plants expressing CsCCD2L gene.

obtain T1 seeds. N. tabacum T1 seeds were plated on hygromycin B (at a final concentration of $50 \mu \mathrm{g} / \mathrm{ml}$ ) selection MS medium. All the lines showed resistance/susceptible segregation under hygromycin selection. T1 and T2 transgenic plants were grown in a greenhouse as described in section "Materials and Methods" (mean irradiance $100 \mu \mathrm{mol} \mathrm{m} \mathrm{m}^{-2} \mathrm{~s}^{-1}$ ) and exhibited a normal phenotype (Figure 2) and were fertile. Based on the segregation patterns of hygromycin-resistant sensitive seedlings, 29 out of the 55 lines were single locus for the introduced transgene. Analysis of genomic DNA confirmed the presence of the intact CsCCD2L cassette.

Ten putative transgenic $N$. glauca plants (T0) were obtained and screened by HPLC-DAD analysis. All of these 10 transgenic T0 lines produced crocins in leaves. After leaf sample collection, 
Wt, and transgenic $N$. glauca T0 plants were grown in a controlled growth chamber with a $25 / 20^{\circ} \mathrm{C}$ day/night temperature regime, a 12 -h photoperiod (mean irradiance $400 \mu \mathrm{mol} \mathrm{m} \mathrm{m}^{-2} \mathrm{~s}^{-1}$ ) and $60-90 \%$ relative humidity. Wt and all the crocin accumulating transgenic $N$. glauca T0 plants were fertile and were selfpollinated to obtain T1 seeds, which took approximately one and a half years in our controlled growth chamber. The transgenic N. glauca T1 seeds were plated on hygromycin B (at a final concentration of $50 \mu \mathrm{g} / \mathrm{ml}$ ) selection MS medium. All the lines showed resistance/susceptible segregation under hygromycin selection. T1 transgenic plants were grown in a greenhouse as described in section "Materials and Methods" (mean irradiance $100 \mu \mathrm{mol} \mathrm{m}{ }^{-2} \mathrm{~s}^{-1}$ ) and exhibited a faint yellow leaf phenotype (Figure 2). Based on the segregation of hygromycin-resistant sensitive seedlings, seven out of the ten lines were deduced to be single locus transgene insertion lines. Analysis of genomic DNA confirmed the presence of the intact CsCCD2L cassette similar to N. tabacum.

\section{Introduction of CsCCD2L, BrCrtZ, and AtOrMut Mutant Genes in the Nicotiana tabacum Genome}

The flower formation of $N$. glauca depends very much on the season, even in a greenhouse. The plant prefers long-day conditions and high intensity of sunlight. Thus, it may take up to 1 year after seedling transfer to the soil before the first flowers set seeds (Gerjets et al., 2007). Therefore, we have chosen the faster growing $N$. tabacum as a model to improve crocin biosynthesis in leaves by transformation with one binary vector carrying CsCCD2L, BrCrtZ, and AtOrMut mutant genes driven by three different promoters (CaMV35S promoter, N. tabacum Ubi.U4 promoter, and Arabidopsis ubiquitin 10 promoters, respectively) (Supplementary Figure 1). Seventy-five putative transgenic N. tabacum T0 plants were generated and screened by crocin analysis in leaves. Thirty-two transgenic plants accumulated crocins in leaves. All the crocin accumulating transgenic N. tabacum T0 plants were fertile and self-pollinated to obtain T1 seeds. T1 seeds were plated on a selection MS medium containing hygromycin B. All 32 lines showed resistance/susceptible segregation for hygromycin selection. T1 and T2 transgenic plants were grown in a greenhouse (mean irradiance $100 \mu \mathrm{mol}$ $\mathrm{m}^{-2} \mathrm{~s}^{-1}$ ) and exhibited a yellow leaf phenotype (Figure 2) and were fertile. Based on the segregation of hygromycin-resistant and hygromycin-sensitive tobacco seedlings on selection MS medium supplemented hygromycin B, 15 out of the 32 lines were deduced to be single-locus transgene insertion lines. The genomic DNA was further verified using PCR to check for the presence of CsCCD2L, BrCrtZ, and AtOrMut mutant transgenes.

\section{Crocin Accumulation in Transgenic Nicotiana glauca and Nicotiana tabacum}

Polar aqueous extracts from seven T1 N. glauca and six T2 $N$. tabacum hygromycin resistant plants were subjected to extraction and analysis for the de novo production of crocins. Identification and quantification of the amounts of crocins were conducted by HPLC-DAD using the method described by

\section{A}

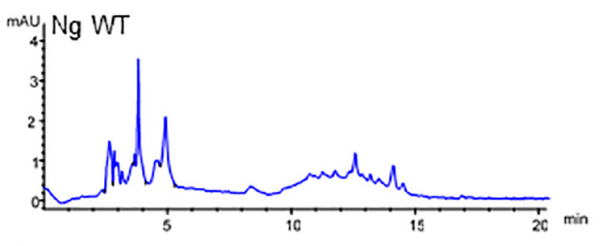

B
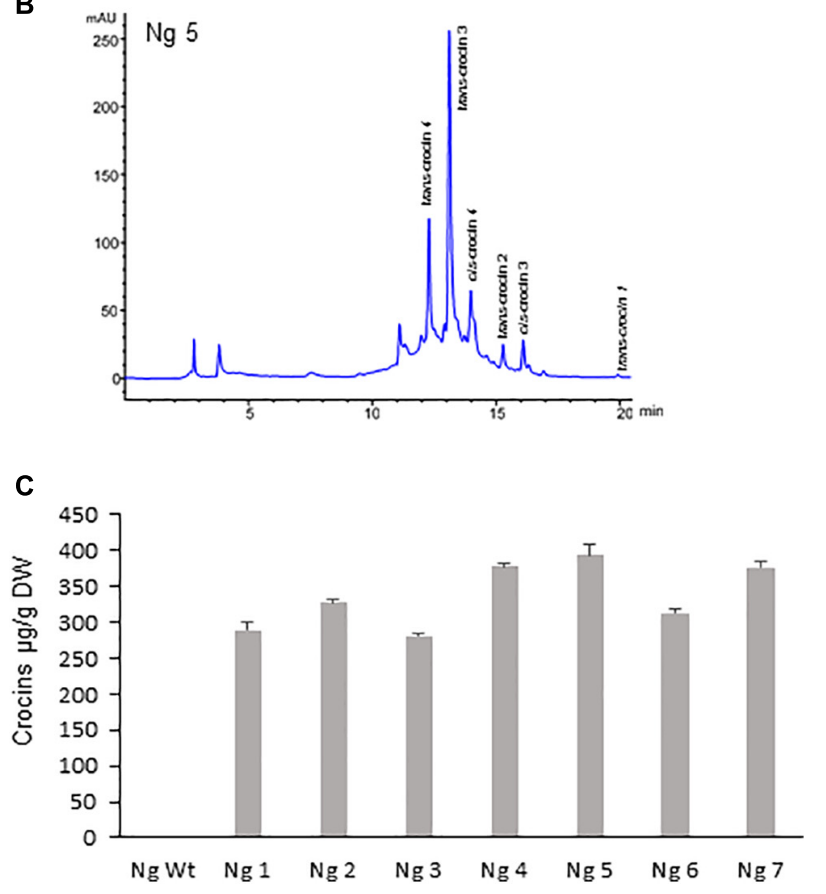

FIGURE 3 | Accumulation of apocarotenoids in Wt and transgenic T1 lines of Nicotiana glauca expressing CsCCD2L. (A) High-performance liquid chromatography-diode array detector (HPLC-DAD) analysis of polar extracts of Wt N. glauca leaves at $440 \mathrm{~nm}$. (B) HPLC-DAD analysis of polar extracts of transgenic $N$. glauca leaves at $440 \mathrm{~nm}$. Note that peaks for the abundant crocins in the transgenic lines (trans-crocin 4, trans-crocin 3, cis-crocin 4, trans-crocin 2, cis-crocin 3, and trans-crocin 1) are completely absent from Wt plants. mAU, milli-absorbance units. (C) Apocarotenoid accumulation in leaves of Wt and transgenic lines. Analyses were done in triplicate. Error bars represent the SD. DW, dry weight.

Diretto et al. (2019). When compared with the chromatogram of the extract of the Wt $N$. glauca (Figures 3A,B) and N. tabacum (Figures $4 \mathbf{A}, \mathbf{B})$ the chromatograms of the transgenic lines showed several peaks with maximum absorbance from 433 to $440 \mathrm{~nm}$. Further analyses of the retentions times and the spectra data compared with the standards for crocins led to the identification of crocins with different degrees of glycosylation, ranging from one to four glucose molecules (Figures 3B, 4B). Such apocarotenoids were absent in the Wt extracts.

Lines from $N$. glauca accumulated a 10-fold higher amount of crocins than N. tabacum lines. Line Ng5 accumulated ca: $400 \mu \mathrm{g} / \mathrm{g}$ DW of crocins (Figures 3C, 4C).

To evaluate whether the introduction of AtOrMut and $\mathrm{BrCrtZ}$ genes in addition to $C s C C D 2 L$ can boost the level of crocins in N. tabacum, polar extracts from seven T2 homozygous lines derived from single-locus transgene insertion lines were 
analyzed to determine the amount of crocins (Figure 5). All the lines accumulated higher levels of crocins as compared with those lines from $N$. tabacum expressing CsCCD2L alone. Interestingly, $N$. glauca transgenic plants accumulated far higher levels of crocins. Line Nt24 was the top line expressing the three transgenes in N. tabacum, accumulating crocins to $136 \mu \mathrm{g} / \mathrm{g}$ DW.

\section{Carotenoid Profiles in Transgenic Nicotiana glauca and Nicotiana tabacum}

Lines accumulating higher levels of crocins were selected for more in-depth analyses. Carotenoids were extracted from four $N$. tabacum lines expressing the three transgenes (CsCCD2L, BrCrtZ, and AtOrMut), three N. glauca lines, and Wt controls and analyzed by HPLC-DAD (Figures 6, 7 and Supplementary Tables 2, 3). In general, the carotenoid content of all transgenic lines was much lower compared to their corresponding $\mathrm{Wt}$ controls. The most prominent differences in the carotenoid composition of the analyzed leaves were for neoxanthin, $\beta$-carotene, and lutein. Neoxanthin, $\beta$-carotene, and lutein levels were substantially decreased in the leaves of transgenic plants compared with Wt (Figures 6C, 7C and Supplementary Tables 2, 3). Changes in the levels of chlorophyll were more pronounced in transgenic plants of $N$. glauca than N. tabacum (Figures 6B, 7B).

\section{Expression Analysis of Transgenes and Selected Endogenous Carotenogenic Genes}

The expression levels of transgenes and selected endogenous carotenoid biosynthetic genes (PSY1, PSY2, LCYB, and BCH) in leaves were monitored by RT-qPCR (Figures $\mathbf{8 A , B}$ ). The expression of CsCCD2L in transgenic N. tabacum plants was much higher than those of $\mathrm{BrCrtZ}$ or AtOrMut genes, implying that the activity of the CaMV35S promoter for CsCCD2L is much stronger than those of tobacco polyubiquitin Ubi.U4 and the Arabidopsis AtUBQ10 promoters for BrCrtZ and AtOrMut genes, respectively. Endogenous carotenoid biosynthetic genes, including phytoene synthase (PSY1 and PSY2) genes, lycopene $\beta$-cyclase $(L C Y B)$ and $\beta$-carotene hydroxylase $(B C H)$ genes, were downregulated in all the transgenic plants as compared with the Wt control (Figures 8A,B).

\section{DISCUSSION}

Crocins are mainly known for being responsible for the color of the saffron spice. In addition to their pigment capacity, crocins show bioactive properties with several therapeutic and pharmacological applications (Mykhailenko et al., 2019). The saffron apocarotenoids had a global market size valued at 374.6 million USD in 2020 and is expected to reach USD 721.5 million by 2028 , being the medical application segment the one projected to be the fastest-growing segment. ${ }^{1}$ Between 120,000 and 200,000 flowers are needed to produce $1 \mathrm{~kg}$ of dried saffron stigma

${ }^{1}$ https://www.grandviewresearch.com

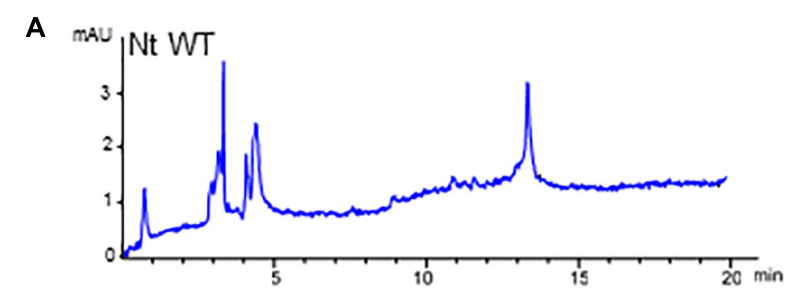

B

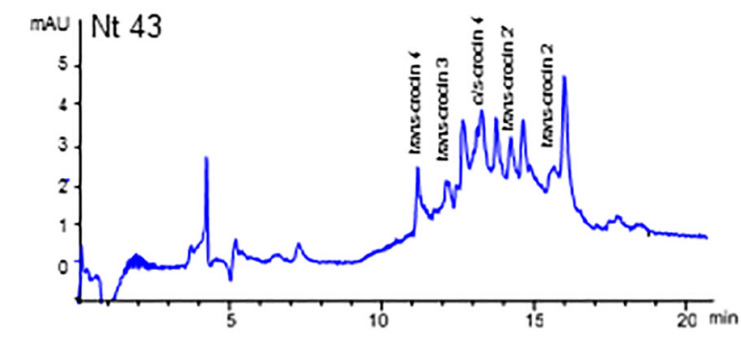

C

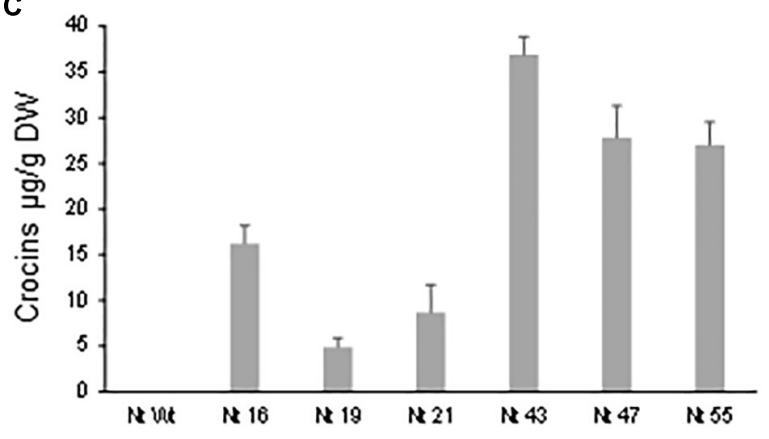

FIGURE 4 | Accumulation of apocarotenoids in wild-type (WT) and transgenic T2 homozygous lines of Nicotiana tabacum expressing CsCCD2L gene. (A) HPLC-DAD analysis of polar extracts of Wt leaves at $440 \mathrm{~nm}$. (B) HPLC-DAD analysis of polar extracts of transgenic leaves at $440 \mathrm{~nm}$. Peaks for the abundant crocins in the transgenic lines (trans-crocin 4 , trans-crocin 3, cis-crocin 4, trans-crocin 2', and trans-crocin 2) are completely absent from Wt plants. mAU, milli-absorbance units. (C) Apocarotenoid accumulation in leaves of Wt and transgenic lines. Analyses were done in triplicate. Error bars represent the SD. DW, dry weight.

threads, which equates to 370-470 h of work. Consequently, the process is very labor-intensive and risky since it is highly dependent on environmental conditions, leading to high costs.

To overcome these limitations for obtaining saffron apocarotenoids while considering the advances of metabolic engineering, its progression into synthetic biology, and the elucidation of the saffron pathway, we were able to engineer plants from both N. glauca and N. tabacum expressing CsCCD2L. Several efforts have been made by the scientific community to transfer the synthesis of apocarotenoids from saffron to other hosts, looking for the low-cost production of these rare metabolites. It is known that the use of the saffron CsCCD2L enzyme resulted in a maximum accumulation of $1.22,15.70$, and $4.42 \mathrm{mg} / \mathrm{l}$ of crocetin in Saccharomyces cerevisiae (Chai et al., 2017; Tan et al., 2019) and Escherichia coli (Wang et al., 2019). In a subsequent study aimed to confirm, in planta, the role of a novel UGTs in picrocrocin biosynthesis (Diretto et al., 2019), 


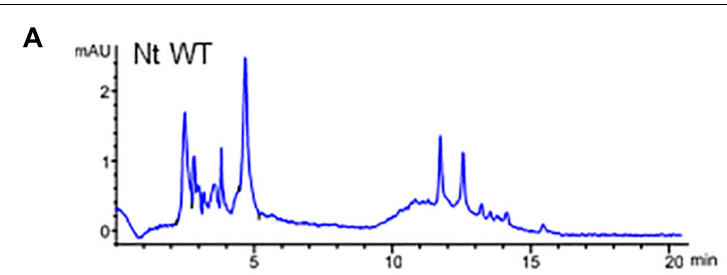

B

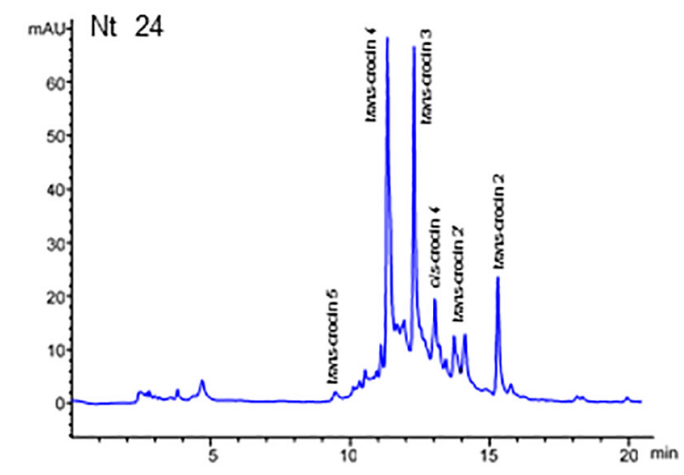

C

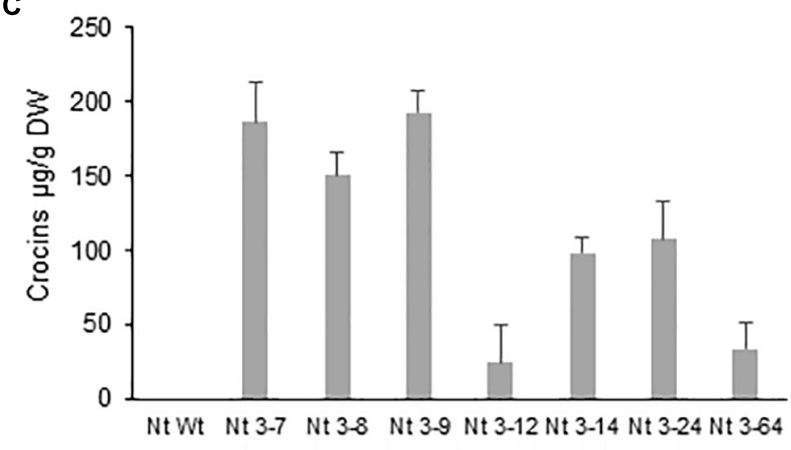

FIGURE 5 | Accumulation of apocarotenoids in wild-type (Wt) and transgenic T2 homozygous lines of Nicotiana tabacum coexpressing CsCCD2L + BrCrtZ + AtOrMut genes. (A) HPLC-DAD analysis of polar extracts of WT leaves at $440 \mathrm{~nm}$. (B) HPLC-DAD analysis of polar extracts of transgenic leaves at $440 \mathrm{~nm}$. Peaks for the abundant crocins in the transgenic lines (trans-crocin 4, trans-crocin 3, cis-crocin 4, trans-crocin 2', and trans-crocin 2) are completely absent from Wt plants. mAU, milli-absorbance units. (C) Apocarotenoid accumulation in leaves of Wt and transgenic lines. Analyses were done in triplicate. Error bars represent the SD. DW, dry weight.

Nicotiana benthamiana leaves were transiently transformed, via A. tumefaciens, with CsCCD2L, alone or in combination with UGT709G1, which led to the production of $30.5 \mu \mathrm{g} / \mathrm{g}$ DW of crocins, with a glycosylation degree ranging from 1 to 4 (crocin 1-4). In addition, Marti et al. (2020), using a virus-driven expression of CsCCD2L in adult $N$. benthamiana plants demonstrated that CsCCD2L expression alone is sufficient for significant crocin accumulation in transiently transformed tobacco (up to $2.18 \mathrm{mg} / \mathrm{g} \mathrm{DW}$ ) (Marti et al., 2020).

The expression of CsCCD2L in $N$. glauca and $N$. tabacum allowed the accumulation of notable amounts of up to 400 and $36 \mu \mathrm{g} / \mathrm{g}$ DW of crocins, respectively. The underlying reason for the accumulated crocin variation observed among the analyzed
N. glauca and N. tabacum lines could be due to the higher transcript levels of CsCCD2L N. glauca lines, and as well to the higher levels of zeaxanthin present in the leaves of $N$. glauca plants. The amount of crocins obtained is much higher in lines from $N$. glauca than in those from $N$. tabacum and the ones previously described using an A. tumefaciens-mediated transient expression of the CsCCD2L in N. benthamiana leaves, alone or in combination with UGT709G1 (30.5 $\mu \mathrm{g} / \mathrm{g}$ DW of crocins). However, this accumulation is lower than the one reported in leaves of adult $N$. benthamiana using a recombinant virus that expressed CsCCD $2 L(2.18 \mathrm{mg} / \mathrm{g} \mathrm{DW}$ of crocins) (Marti et al., 2020). However, crocin production in N. gluaca provides be more effective compared to the data obtained for the production of other carotenoids in this plant; expression of the $\beta$-carotene ketolase $(\mathrm{CrtO})$ gene led to a total ketocarotenoid concentration in leaves of 136.6 (young) or 156.1 (older) $\mu \mathrm{g} / \mathrm{g}$ dry weight and in petals of 165 Mg/g dry weight (Zhu et al., 2007; Mortimer et al., 2017).

The profiles of crocins were different from those reported in saffron stigma, where trans-crocin 4, followed by transcrocin 3 were the major crocins detected. In contrast, in our transgenic plants, trans-crocin-3 was more abundant than trans-crocin-4. In $N$. benthamiana leaves expressing transiently only $C s C C D 2 L$, the major crocin species were the trans-crocin 3, and trans-crocin with two glucose molecules (Supplementary Figure 3). These data indicated that different endogenous UGTs belonging to Nicotiana species recognize crocetin and crocins and can transfer different glucose molecules by changing the qualitative pattern. However, other factors, such as the presence of hydrolase activities that affect the stability of the different crocins synthesized in tobacco cells, and the transport of these crocins to the vacuole and their stability therein, could be influencing the different profiles observed.

It is well-known that $B C H$ overexpression resulted in increased zeaxanthin and xanthophyll contents, both in microbes and plants (Lagarde et al., 2000; Du et al., 2010; Arango et al., 2014). Therefore, overexpression of $B C H$ could enhance the levels of zeaxanthin as the preferred substrate of CsCCD2L (Frusciante et al., 2014). On the other hand, the Orange gene has proven to play an important role in carotenoid accumulation by activating chromoplast differentiation in non-green tissues ( $\mathrm{Lu}$ et al., 2006), although its expression in green tissues exerts no effect on carotenoid levels (Yuan et al., 2015), we decided to determine whether its introduction could increase the carotenoid content by promoting the formation of carotenoid-sequestering structures. In this context, $\mathrm{BrCrtZ}$ and AtOrMut were introduced in N. tabacum to boost the crocin content, since the accumulation of these metabolites was 10 -fold lower than $N$. glauca. The introduction of the two transgenes together with $C s C C D 2 L$ has most likely led to an increase in the carotenoid pool used as a substrate by the enzyme and, consequently, in a 3.5fold increase in crocin compared to the sole expression of CsCCD2L.

By introducing CsCCD2L alone or in combination with BrCrtZ and AtOrMut, a decreased level of the major and 


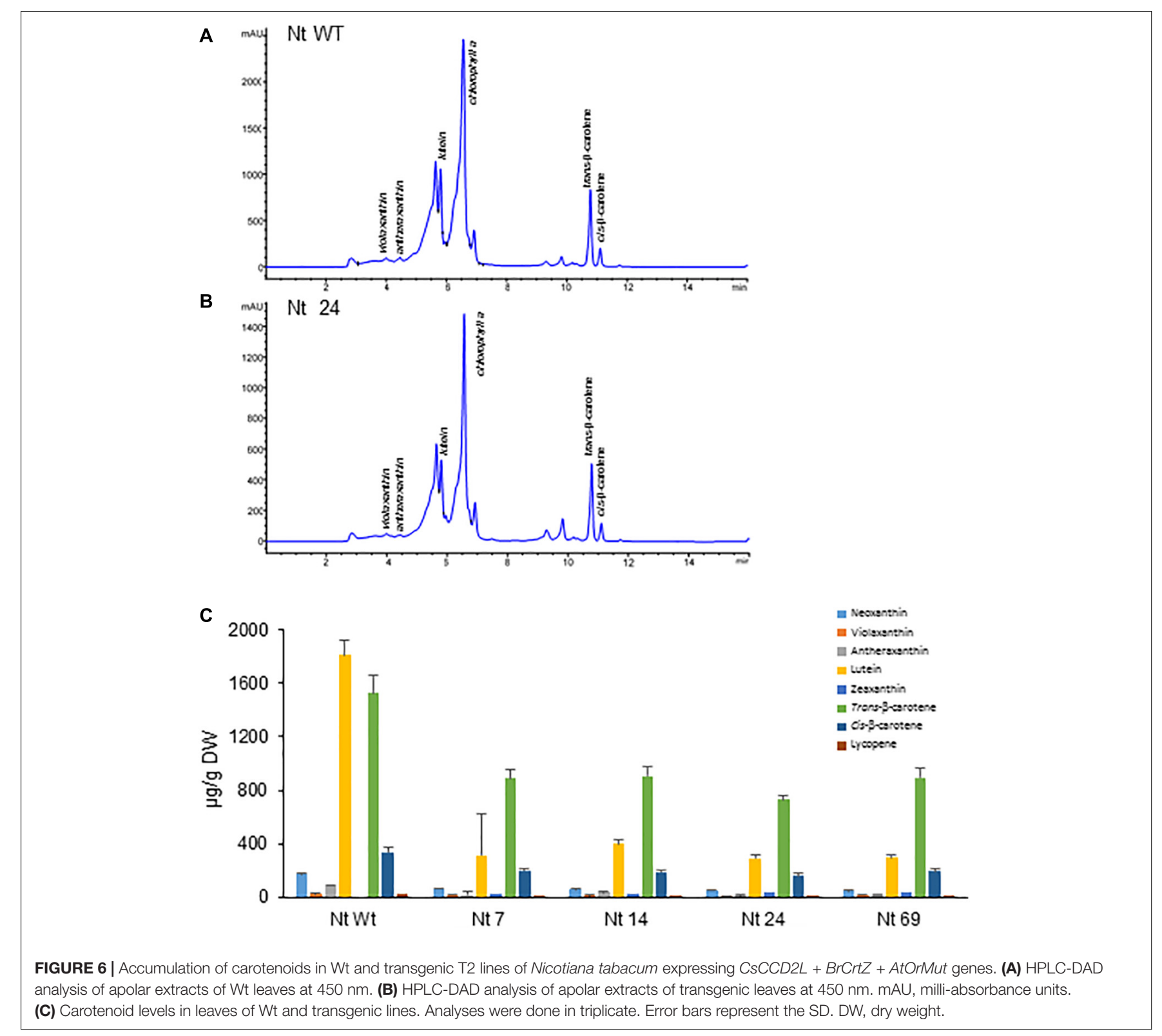

other minor carotenoids normally present in N. glauca and $N$. tabacum was detected. Among all the carotenoids detected, lutein was one of the carotenoids showing the higher decrease, likely due to the ability of $C s C C D 2 L$ to act over the $\beta$-ring of this molecule as reported in previous in vitro studies where lutein can act as a substrate of this cleavage activity (Frusciante et al., 2014). Zeaxanthin, the crocin precursor, is not a major carotenoid in Nicotiana plant leaves, although the production of the crocins in N. glauca and N. tabacum suggests that the expression of CsCCD2L can drag the leaf metabolic flux toward the production of these apocarotenoids. The level of $\beta$-carotene was also lower among the N. glauca transgenic plants, the same phenomenon has been shown in N. tabacum plants transformed with the three genes suggesting a hydroxylation of $\beta$-carotene to zeaxanthin under the influence of CsCCD2L in $N$. glauca and the combination of the exogenous $B r C r t Z$ and $C s C C D 2 L$ in $N$. tabacum. Gotz et al. (2002), previously described the increase of zeaxanthin in tobacco plants (N. tabacum L. cv. Samsun) by transformation with a heterologous carotenoid gene encoding $\beta$-carotene hydroxylase (CrtZ) from Erwinia uredovora under constitutive promoter control. The increase of zeaxanthin in transgenic plants is due to the catalytic activity of the additional $\beta$-carotene hydroxylase rather than being primarily caused by an elevation in the conversion of violaxanthin to zeaxanthin in high light (Gotz et al., 2002).

It has been reported that the expression of an appropriate CsCCD2L in $N$. benthamiana is sufficient to activate the apocarotenoid pathway in this plant (Diretto et al., 2019; Marti et al., 2020). It seems that both $N$. glauca and $N$. tabacum followed the same pattern as $N$. benthamiana. The crocins 


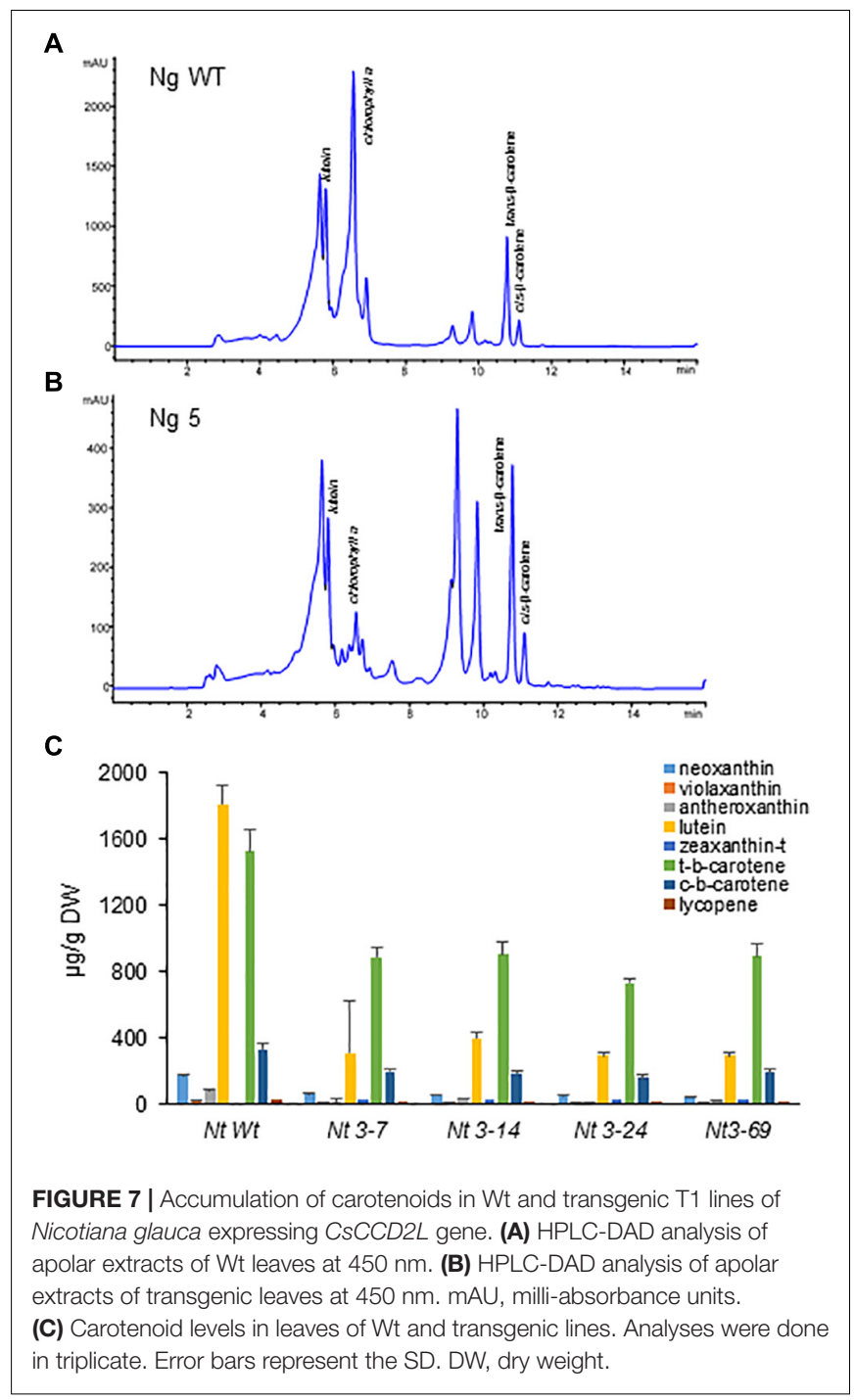

produced in this report, using metabolically engineered $N$. glauca (400 $\mu \mathrm{g} / \mathrm{g} \mathrm{DW}$ ) and N. tabacum (136 $\mu \mathrm{g} / \mathrm{g} \mathrm{DW}$ ), are lower than those obtained in $N$. benthamiana $(2 \mathrm{mg} / \mathrm{g} \mathrm{DW})$ using a virus-driven system expressing transient CsCCD2L. However, the stable expression of $C s C C D 2 L$ can compensate for the low apocarotenoid yields leading to higher apocarotenoid productivities by reducing costs and labor. Further increase of N. glauca and N. tabacum content in these apocarotenoids can also be achieved by combining different types of promoters and the expression of other carotenogenic genes to increase the content of the precursors of crocins, zeaxanthin, and lutein. Thus, an approach of focused metabolic engineering intended toward the production of crocins will establish $N$. glauca as a potential industrial host for the production of these apocarotenoids. N. glauca is not only a better system for crocin biosynthesis and accumulation than $N$. tabacum but also a nicotine-free species and perennial plant from which the leafy biomass can be harvested repeatedly for crocin production.
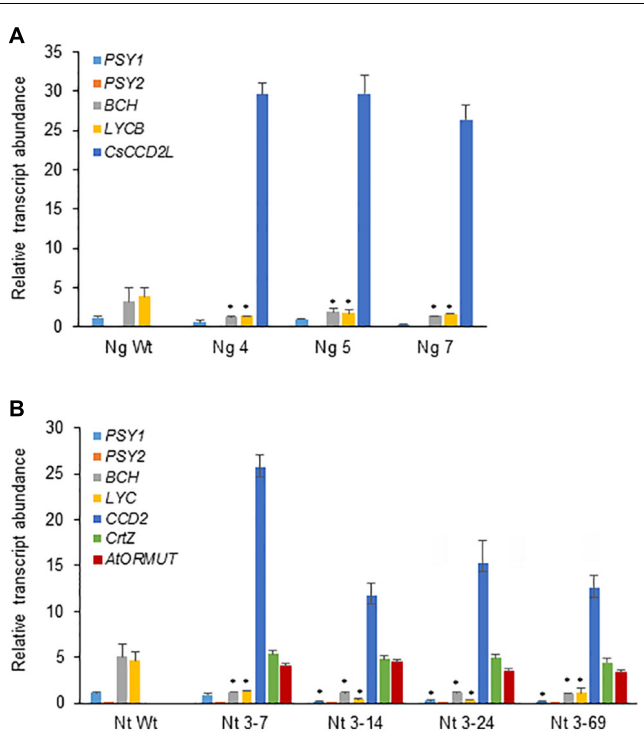

FIGURE 8 | Relative transcript abundance of different transcripts in Nicotiana glauca (A) and N. tabacum (B), wild-type $(\mathrm{Wt})$ and selected transgenic lines for crocin accumulation ( $\mathrm{Ng}$ lines expressing CsCCD2L gene; Nt lines coexpressing CsCCD2L, BrCrtZ, and AtOrMut genes). RT-qPCR was used to quantify gene expression levels in three biological replicates per sample. Error bars represent the SD. The asterisk above the bars indicates $P$-values (Student's $t$-test) statistically significant $(P<0.05)$.

\section{DATA AVAILABILITY STATEMENT}

The original contributions presented in the study are included in the article/Supplementary Material, further inquiries can be directed to the corresponding author/s.

\section{AUTHOR CONTRIBUTIONS}

CZ, OA, PC, and LG-G conceived, designed the research, and wrote the manuscript. CZ, OA, XH, AR-M, TC, and LG-G conducted the experiments. CZ, OA, XH, AR-M, TC, PC, and LG-G analyzed the data. All authors read and approved the final version of the manuscript.

\section{FUNDING}

This study was supported by grants BIO2016-77000-R from the Spanish Ministerio de Ciencia; Innovación $\mathrm{y}$ Universidades and SBPLY/17/180501/000234 from the Junta de Comunidades de Castilla-La Mancha (co-financed European Union FEDER funds), the National Natural Science Foundation of China (31870278), the Spanish Ministry of Economy and Competitiveness (MINECO), Spain (RTI2018097613-B-I00 and PGC2018-097655-B-I00). CZ and LG-G were participants of the European COST action CA15136 (EUROCAROTEN), and Programa Estatal de Investigación Científica y Técnica de Excelencia, Spain (BIO2015-71703-REDT and BIO2017-90877-REDT). 


\section{ACKNOWLEDGMENTS}

We thank J. Argandoña at the University of Castilla-LA Mancha, for providing technical assistance. We also thank K. Walsh for the language editing of the manuscript.

\section{REFERENCES}

Ahrazem, O., Diretto, G., Argandona, J., Rubio-Moraga, A., Julve, J. M., Orzaez, D., et al. (2017). Evolutionarily distinct carotenoid cleavage dioxygenases are responsible for crocetin production in Buddleja davidii. J. Exp. Bot. 68, 46634677. doi: 10.1093/jxb/erx277

Ahrazem, O., Gomez-Gomez, L., Rodrigo, M. J., Avalos, J., and Limon, M. C. (2016a). Carotenoid cleavage oxygenases from microbes and photosynthetic organisms: features and functions. Int. J. Mol. Sci. 17:781. doi: 10.3390/ ijms 17111781

Ahrazem, O., Rubio-Moraga, A., Argandona-Picazo, J., Castillo, R., and GomezGomez, L. (2016b). Intron retention and rhythmic diel pattern regulation of carotenoid cleavage dioxygenase 2 during crocetin biosynthesis in saffron. Plant Mol. Biol. 91, 355-374. doi: 10.1007/s11103-016-0473-8

Ahrazem, O., Rubio-Moraga, A., Berman, J., Capell, T., Christou, P., Zhu, C., et al. (2016c). The carotenoid cleavage dioxygenase CCD2 catalysing the synthesis of crocetin in spring crocuses and saffron is a plastidial enzyme. New Phytol. 209, 650-663. doi: 10.1111/nph.13609

Ahrazem, O., Rubio-Moraga, A., Nebauer, S. G., Molina, R. V., and GomezGomez, L. (2015). Saffron: its phytochemistry, developmental processes, and biotechnological prospects. J. Agric. Food Chem. 63, 8751-8764. doi: 10.1021/ acs.jafc.5b03194

Amin, B., and Hosseinzadeh, H. (2012). Evaluation of aqueous and ethanolic extracts of saffron, Crocus sativus L., and its constituents, safranal and crocin in allodynia and hyperalgesia induced by chronic constriction injury model of neuropathic pain in rats. Fitoterapia 83, 888-895. doi: 10.1016/j.fitote.2012.03. 022

Arango, J., Jourdan, M., Geoffriau, E., Beyer, P., and Welsch, R. (2014). Carotene hydroxylase activity determines the levels of both alpha-carotene and total carotenoids in orange carrots. Plant Cell. 26, 2223-2233. doi: 10.1105/tpc.113. 122127

Bukhari, S. I., Manzoor, M., and Dhar, M. K. (2018). A comprehensive review of the pharmacological potential of Crocus sativus and its bioactive apocarotenoids. Biomed. Pharmacother. 98, 733-745. doi: 10.1016/j.biopha.2017.12.090

Chai, F., Wang, Y., Mei, X., Yao, M., Chen, Y., Liu, H., et al. (2017). Heterologous biosynthesis and manipulation of crocetin in Saccharomyces cerevisiae. Microb. Cell Fact. 16, 54. doi: 10.1186/s12934-017-0665-1

Christodoulou, E., Kadoglou, N. P., Kostomitsopoulos, N., and Valsami, G. (2015). Saffron: a natural product with potential pharmaceutical applications. J. Pharm. Pharmacol. 67, 1634-1649. doi: 10.1111/jphp.12456

Diretto, G., Ahrazem, O., Rubio-Moraga, A., Fiore, A., Sevi, F., Argandona, J., et al. (2019). UGT709G1: a novel uridine diphosphate glycosyltransferase involved in the biosynthesis of picrocrocin, the precursor of safranal in saffron (Crocus sativus). New Phytol. 224, 725-740. doi: 10.1111/nph.16079

Diretto, G., López-Jiménez, A. J., Ahrazem, O., Frusciante, S., Song, J., RubioMoraga, A., et al. (2021). Identification and characterization of apocarotenoid modifiers and carotenogenic enzymes for biosynthesis of crocins in Buddleja davidii flowers. J. Exp. Bot. 72, 3200-3218. doi: 10.1093/jxb/erab053

Du, H., Wang, N., Cui, F., Li, X., Xiao, J., and Xiong, L. (2010). Characterization of the beta-carotene hydroxylase gene DSM2 conferring drought and oxidative stress resistance by increasing xanthophylls and abscisic acid synthesis in rice. Plant Physiol. 154, 1304-1318. doi: 10.1104/pp.110.163741

Eroglu, A., and Harrison, E. H. (2013). Carotenoid metabolism in mammals, including man: formation, occurrence, and function of apocarotenoids. J. Lipid Res. 54, 1719-1730. doi: 10.1194/jlr.R039537

Farre, G., Blancquaert, D., Capell, T., Van Der Straeten, D., Christou, P., and Zhu, C. (2014). Engineering complex metabolic pathways in plants. Annu. Rev. Plant Biol. 65, 187-223. doi: 10.1146/annurev-arplant-050213-035825

Fiedor, J., and Burda, K. (2014). Potential role of carotenoids as antioxidants in human health and disease. Nutrients 6, 466-488. doi: 10.3390/nu6020466

\section{SUPPLEMENTARY MATERIAL}

The Supplementary Material for this article can be found online at: https://www.frontiersin.org/articles/10.3389/fpls.2022. 861140/full\#supplementary-material

Finley, J. W., and Gao, S. (2017). A perspective on crocus sativus 1. (saffron) constituent crocin: a potent water-soluble antioxidant and potential therapy for Alzheimer's Disease. J. Agric. Food Chem. 65, 1005-1020. doi: 10.1021/acs.jafc. $6 \mathrm{~b} 04398$

Fraser, P. D., and Bramley, P. M. (2004). The biosynthesis and nutritional uses of carotenoids. Prog. Lipid Res. 43, 228-265. doi: 10.1016/j.plipres.2003.10.002

Frusciante, S., Diretto, G., Bruno, M., Ferrante, P., Pietrella, M., Prado-Cabrero, A., et al. (2014). Novel carotenoid cleavage dioxygenase catalyzes the first dedicated step in saffron crocin biosynthesis. Proc. Natl. Acad. Sci. USA 111, 12246-12251. doi: 10.1073/pnas.1404629111

Georgiadou, G., Tarantilis, P. A., and Pitsikas, N. (2012). Effects of the active constituents of Crocus Sativus L., crocins, in an animal model of obsessivecompulsive disorder. Neurosci. Lett. 528, 27-30. doi: 10.1016/j.neulet.2012.08. 081

Gerjets, T., Sandmann, M., Zhu, C., and Sandmann, G. (2007). Metabolic engineering of ketocarotenoid biosynthesis in leaves and flowers of tobacco species. Biotechnol. J. 2, 1263-1269. doi: 10.1002/biot.200700040

Gotz, T., Sandmann, G., and Romer, S. (2002). Expression of a bacterial carotene hydroxylase gene (crtZ) enhances UV tolerance in tobacco. Plant Mol. Biol. 50, 129-142. doi: 10.1023/a:1016072218801

Horsch, R. B., Rogers, S. G. and Fraley, R. T. (1985). Transgenic plants. Cold Spring Harb. Symp. Quant. Biol. 50, 433-437. doi: 10.1101/sqb.1985.050.01.054

Jia, K. P., Baz, L., and Al-Babili, S. (2017). From Carotenoids to Strigolactones. J. Exp. Bot. 2017:476. doi: 10.1093/jxb/erx476

Lagarde, D., Beuf, L., and Vermaas, W. (2000). Increased production of zeaxanthin and other pigments by application of genetic engineering techniques to Synechocystis sp. strain PCC 6803. Appl. Environ. Microbiol. 66, 64-72. doi: 10.1128/AEM.66.1.64-72.2000

Liao, Y. H., Houghton, P. J., and Hoult, J. R. (1999). Novel and known constituents from Buddleja species and their activity against leukocyte eicosanoid generation. J. Nat. Prod. 62, 1241-1245. doi: 10.1021/np990092+

López, A. J., Frusciante, S., Niza, E., Ahrazem, O., Rubio-Moraga, A., Diretto, G., et al. (2021). A New glycosyltransferase enzyme from family 91, ugt91p3, is responsible for the final glucosylation step of crocins in saffron (Crocus sativus L.). Int. J. Mol. Sci. 22:8815. doi: 10.3390/ijms22168815

Lopresti, A. L., and Drummond, P. D. (2014). Saffron (Crocus sativus) for depression: a systematic review of clinical studies and examination of underlying antidepressant mechanisms of action. Hum. Psychopharmacol. 29, 517-527. doi: 10.1002/hup.2434

Lu, S., Van Eck, J., Zhou, X., Lopez, A. B., O’Halloran, D. M., Cosman, K. M., et al. (2006). The cauliflower Or gene encodes a DnaJ cysteine-rich domaincontaining protein that mediates high levels of beta-carotene accumulation. Plant Cell. 18, 3594-3605. doi: 10.1105/tpc.106.046417

Marti, M., Diretto, G., Aragones, V., Frusciante, S., Ahrazem, O., Gomez-Gomez, L., et al. (2020). Efficient production of saffron crocins and picrocrocin in Nicotiana benthamiana using a virus-driven system. Metab. Eng. 61, 238-250. doi: 10.1016/j.ymben.2020.06.009

Mazidi, M., Shemshian, M., Mousavi, S. H., Norouzy, A., Kermani, T., Moghiman, T., et al. (2016). A double-blind, randomized and placebocontrolled trial of Saffron (Crocus sativus L.) in the treatment of anxiety and depression. J. Compl. Integr. Med. 13, 195-199. doi: 10.1515/jcim-201 5-0043

Molina-Hidalgo, F. J., Vazquez-Vilar, M., D’Andrea, L., Demurtas, O. C., Fraser, P., Giuliano, G., et al. (2021). Engineering metabolism in nicotiana species: a promising future. Trends Biotechnol. 39, 901-913. doi: 10.1016/j.tibtech.2020. 11.012

Moraga, A. R., Nohales, P. F., Perez, J. A., and Gomez-Gomez, L. (2004). Glucosylation of the saffron apocarotenoid crocetin by a glucosyltransferase isolated from Crocus sativus stigmas. Planta 219, 955-966. doi: 10.1007/s00425004-1299-1 
Moraga, A. R., Rambla, J. L., Ahrazem, O., Granell, A., and Gomez-Gomez, L. (2009). Metabolite and target transcript analyses during Crocus sativus stigma development. Phytochemistry 70, 1009-1016. doi: 10.1016/j.phytochem.2009. 04.022

Moras, B., Loffredo, L., and Rey, S. (2018). Quality assessment of saffron (Crocus sativus L.) extracts via UHPLC-DAD-MS analysis and detection of adulteration using gardenia fruit extract (Gardenia jasminoides Ellis). Food Chem. 257, 325-332. doi: 10.1016/j.foodchem.2018.03.025

Mortimer, C. L., Misawa, N., Perez-Fons, L., Robertson, F. P., Harada, H., Bramley, P. M., et al. (2017). The formation and sequestration of nonendogenous ketocarotenoids in transgenic nicotiana glauca. Plant Physiol. 173, 1617-1635. doi: 10.1104/pp.16.01297

Mykhailenko, O., Kovalyov, V., Goryacha, O., Ivanauskas, L., and Georgiyants, V. (2019). Biologically active compounds and pharmacological activities of species of the genus Crocus: A review. Phytochemistry 162, 56-89. doi: 10.1016/ j.phytochem.2019.02.004

Nagatoshi, M., Terasaka, K., Owaki, M., Sota, M., Inukai, T., Nagatsu, A., et al. (2012). UGT75L6 and UGT94E5 mediate sequential glucosylation of crocetin to crocin in Gardenia jasminoides. FEBS Lett. 586, 1055-1061. doi: 10.1016/j. febslet.2012.03.003

Nam, K. N., Park, Y. M., Jung, H. J., Lee, J. Y., Min, B. D., Park, S. U., et al. (2010). Anti-inflammatory effects of crocin and crocetin in rat brain microglial cells. Eur. J. Pharmacol. 648, 110-116. doi: 10.1016/j.ejphar.2010.09.003

Pfister, S., Steck, A., and Pfander, H. (1996). Isolation and structure elucidation of carotenoid glycoslyesters on gardenia fruits (Gardenia jasminoides) and saffron (Crocus sativus). J. Agricult. Food Chem. 44, 2612-2615. doi: 10.1021/ JF950713E

Sarrion-Perdigones, A., Palaci, J., Granell, A., and Orzaez, D. (2014). Design and construction of multigenic constructs for plant biotechnology using the GoldenBraid cloning strategy. Methods Mol. Biol. 1116, 133-151. doi: 10.1007/ 978-1-62703-764-8_10

Sarrion-Perdigones, A., Vazquez-Vilar, M., Palaci, J., Castelijns, B., Forment, J., Ziarsolo, P., et al. (2013). GoldenBraid 2.0: a comprehensive DNA assembly framework for plant synthetic biology. Plant Physiol. 162, 1618-1631. doi: 10. 1104/pp.113.217661

Skladnev, N. V., and Johnstone, D. M. (2017). Neuroprotective properties of dietary saffron: more than just a chemical scavenger? Neural. Regen. Res. 12, 210-211. doi: 10.4103/1673-5374.198976

Tan, H., Chen, X., Liang, N., Chen, R., Chen, J., Hu, C., et al. (2019). Transcriptome analysis reveals novel enzymes for apo-carotenoid biosynthesis in saffron and allows construction of a pathway for crocetin synthesis in yeast. J. Exp. Bot. 70, 4819-4834. doi: 10.1093/jxb/erz211
Tarantilis, P. A., Tsoupras, G., and Polissiou, M. (1995). Determination of saffron (Crocus sativus L.) components in crude plant extract using highperformance liquid chromatography-UV-visible photodiode-array detectionmass spectrometry. J. Chromatogr. 699, 107-118. doi: 10.1016/0021-9673(95) 00044-n

Walter, M. H., Floss, D. S., and Strack, D. (2010). Apocarotenoids: hormones, mycorrhizal metabolites and aroma volatiles. Planta 232, 1-17. doi: 10.1007/ s00425-010-1156-3

Wang, W., He, P., Zhao, D., Ye, L., Dai, L., Zhang, X., et al. (2019). Construction of Escherichia coli cell factories for crocin biosynthesis. Microb. Cell Fact. 18:120. doi: 10.1186/s12934-019-1166-1

Xu, Z., Pu, X., Gao, R., Demurtas, O. C., Fleck, S. J., Richter, M., et al. (2020). Tandem gene duplications drive divergent evolution of caffeine and crocin biosynthetic pathways in plants. BMC Biol. 18:63. doi: 10.1186/s12915-02000795-3

Yuan, H., Owsiany, K., Sheeja, T., Zhou, X., Rodriguez, C., Li, Y., et al. (2015). A single amino acid substitution in an ORANGE protein promotes carotenoid overaccumulation in Arabidopsis. Plant Physiol. 169, 421-431. doi: 10.1104/pp. 15.00971

Zhu, C., Gerjets, T., and Sandmann, G. (2007). Nicotiana glauca engineered for the production of ketocarotenoids in flowers and leaves by expressing the cyanobacterial crtO ketolase gene. Transgenic. Res. 16, 813-821. doi: 10.1007/ s11248-007-9151-6

Conflict of Interest: The authors declare that the research was conducted in the absence of any commercial or financial relationships that could be construed as a potential conflict of interest.

Publisher's Note: All claims expressed in this article are solely those of the authors and do not necessarily represent those of their affiliated organizations, or those of the publisher, the editors and the reviewers. Any product that may be evaluated in this article, or claim that may be made by its manufacturer, is not guaranteed or endorsed by the publisher.

Copyright (c) 2022 Ahrazem, Zhu, Huang, Rubio-Moraga, Capell, Christou and Gómez-Gómez. This is an open-access article distributed under the terms of the Creative Commons Attribution License (CC BY). The use, distribution or reproduction in other forums is permitted, provided the original author(s) and the copyright owner(s) are credited and that the original publication in this journal is cited, in accordance with accepted academic practice. No use, distribution or reproduction is permitted which does not comply with these terms. 